\title{
On MIMO Channel Modeling for the Mobile Wireless Systems
}

\author{
Rachna Mahey and Dr. Jyoteesh Malhotra \\ ECE Department, Guru Nanak Dev University, Regional Campus, Jalandhar, \\ INDIA \\ rachnamaheyr@gmail.com,jyoteesh@gmail.com
}

\begin{abstract}
Developments in the area of wireless communications have constantly aimed at providing higher data rates even under complex environments using multiple-input multiple-output (MIMO) systems. Hence it generates a huge demand to investigate and understand the MIMO channel models for mobile wireless systems. Channel models describe the communication environments and are necessary in developing efficient wireless communication networks. This paper surveys the different channel models used to characterize the wireless systems. Generally, the channel models are classified as Physical models and Analytical models. These models are analyzed here along with the discussion of popular examples from both model types. The MIMO channel models employed in the current standardization activities to evaluate the MIMO system are also reviewed. Finally, some issues regarding these channel models are highlighted.
\end{abstract}

Keywords: MIMO Channel Modeling, Physical Models, Analytical Models, Standardized Models

\section{Introduction}

Multiple Input Multiple Output (MIMO) systems have recently become one of the most active research topics in the field of wireless communications. New wireless standards are using MIMO as their PHY layer technology because these wireless systems require high data rates which are promising by the MIMO systems. MIMO provides array gain, diversity gain, spatial multiplexing gain and interference reduction gain. MIMO technologies offer significant advantages over single antenna systems. These advantages include extended range, improved reliability in fading environments and higher data throughputs. Channel capacity of a multi-antenna system is increased as compared to a conventional single antenna systems, without any additional transmit power or spectral bandwidth. It is the part of modern wireless communication standards such as IEEE 802.11n (Wi-Fi), MBWA, HSPA, WiMAX-m and LTE Advanced [1]. MIMO systems basically use multiple antenna elements at both the transmitter and the receiver. They utilize independent multipath propagation and channel fading to increase the capacity. MIMO systems can be used to either send the same bit stream from all the transmit antennas and using the Spatial Diversity gain for reliable communication or by sending independent bit streams from the transmit antennas, as in Spatial Multiplexing, to increase the data transmission rate. For the study of these MIMO systems, simple and accurate channel models are required to represent the wireless channel [2].

In general, MIMO channel models are important for the optimization, test, and performance evaluation of wireless communication systems. As the propagation conditions determine the channel characteristics, and the channel capacity of a MIMO system, therefore it is important to develop the MIMO channel models to enable studying the impact of the channel parameters on the capacity of MIMO channels in different propagation environments [3]. Thus the challenge facing communication engineers is to 
develop realistic channel models that can efficiently and accurately predict the performance of a wireless system [4].

This paper provides a survey on the most significant developments in the field of MIMO channel modeling. Several different classes of MIMO channel models are summarized, here. Firstly, a simple MIMO system model is described in Section 2. The different channel models classifications are briefly discussed in Section. A significant categorization of MIMO channel models is the Physical models and Analytical models, which are then reviewed in Section 4 along with the Standardized channel models; then various issues found in the literature are extracted and discussed in Section 5; and finally, some open issues are presented in Section 6.

\section{MIMO Propagation Model}

In contrast to conventional single antenna communication systems, the MIMO systems are equipped with multiple antennas at both the transmitter and receiver ends. Therefore, the MIMO channel has to be expressed for all transmit and receive antenna pairs. Consider $\mathrm{N}_{T} \times \mathrm{N}_{\mathrm{R}}$ MIMO systems with $\mathrm{N}_{\mathrm{T}}$ transmit and $\mathrm{N}_{\mathrm{R}}$ receive antennas, as shown in Figure 1. From a system level viewpoint, a linear time-variant MIMO channel is represented by an $\mathrm{N}_{\mathrm{T}} \times \mathrm{N}_{\mathrm{R}}$ channel matrix [5].

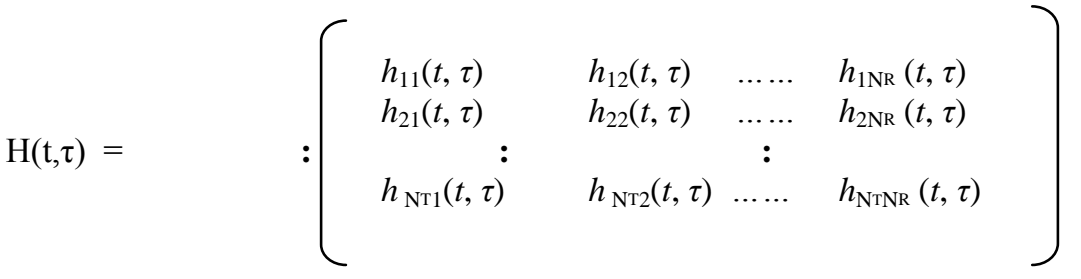

Here $\mathrm{h}_{\mathrm{ij}}(\mathrm{t}, \tau)$ is the time-variant impulse response between the $\mathrm{j}^{\text {th }}$ transmit and $\mathrm{i}^{\text {th }}$ receive antenna. The characteristic of the MIMO channel is determined by the distribution of the elements of channel matrix $\mathrm{H}$, which in turn varies according to the underlying physical propagation environment. For instance, the elements of $\mathrm{H}$ can follow Rayleigh distribution, Rician distribution or Nakagami distribution. The channel matrix (eq. (1)) includes the effects of antennas, its type and configuration, etc.; along with the frequency filtering. It can be used to formulate an over-all MIMO input-output relation between the transmit signal vector $\mathrm{s}(\mathrm{t})$ of length- $\mathrm{N}_{\mathrm{R}}$ and the receive signal vector $\mathrm{y}(\mathrm{t})$ of length- $\mathrm{N}_{\mathrm{T}}$ as [6]:

$$
\mathrm{y}(\mathrm{t})=\mathrm{H}(\mathrm{t}, \tau) \mathrm{s}(\mathrm{t}-\tau) \mathrm{d} \tau+\mathrm{n}(\mathrm{t})
$$

Here, $n(t)$ represents the noise and interference. The dependence of the channel matrix on $t$ vanishes (i.e. $H(\tau)=H(t, \tau)$ ) if the channel is time-invariant. If the bandwidth of signal is sufficiently narrow such that the channel is considered to be approximately constant over the operating frequency, i.e. frequency flat channel, then the corresponding inputoutput relationship in eq. (2) simplifies to as [6]:

$$
y(t)=H s(t)+n(t)
$$




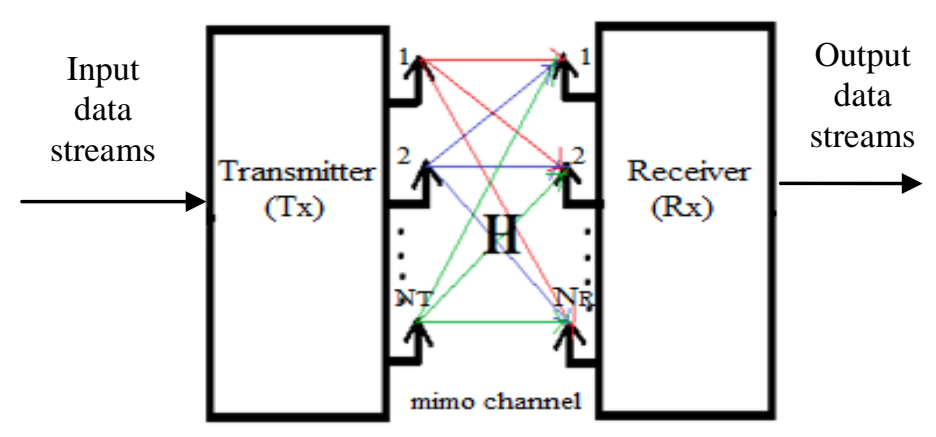

\section{Figure 1. MIMO System with $\mathrm{N}_{\mathrm{T}}$ Transmit Antennas And $\mathrm{N}_{\mathrm{R}}$ Receive Antennas [7]}

The modeling of channel impulse response $\mathrm{h}(\mathrm{t})$ or the channel matrix $\mathrm{H}$ is significant for the simulations of MIMO communication systems. Moreover, the elements of the MIMO channel matrix are not always independent, because of the insufficient antenna elements spacing and the limited environmental scattering.

\section{Channel Model Classification}

Various different forms of MIMO channel models have been described through the past years. Depending on the type of channel considered, there are different classifications from the channel modeling, such as; Field Measurements vs. Scatterer Models Field Measurements, Wideband Models vs. Narrowband Models, and Physical Models vs. Nonphysical Models.

To model the MIMO channels, one approach is to scale the MIMO channel responses during the field metrics. So the channels may be classified into the field measurements model and scatterer field measurements models. Field Measurements Model measures the MIMO channel responses through field measurements. Means some of the important characteristics of MIMO channel can be obtained through investigating the registered data. Scatterer Field Measurements Models (usually involve distributed scatterers) capture the channel characteristics. Hence this model may be utilized to explain the necessary characteristics for the MIMO channel while the built scattering environment is sensible [8].

By looking at the bandwidth of the system, the MIMO channel models may be split into the wideband models and the narrowband models [9] The Wideband Models consider the propagation channel as frequency selective, thus different frequency sub-bands have different channel responses [8]. For Narrowband Models the channel is assumed to have frequency non-selective fading, hence the channel has the same frequency response over the entire system bandwidth [9].

The MIMO channel models may be split into the physical and analytical models [6]. The Physical Models consider some important physical parameters, like AOA (angle of arrival), AOD (angle of departure) and TOA (time of arrival), to describe the MIMO channels. This model describes the characteristics of MIMO channel and the surrounding scattering environment, reasonably [8]. The Non-Physical Models (or Analytical channel models) are derived from statistical characteristics of MIMO channel [2]. In contrast to the physical models, the analytical models represent the impulse response or equivalently the transfer function for the channel between the individually transmit $\mathrm{Tx}$ and receive $\mathrm{Tr}$ antenna array will be described in a mathematical way. Analytical models are comparatively easy to simulate but they could give a limited insight to the MIMO channel propagation characteristics. 


\section{Literature Survey}

Hereafter, this paper is focused on the mostly used MIMO channel model classification. The different types of this channel classification of are summarized, starting with the Physical and Analytical channel models, leading to the Standardized MIMO channel models. An outline of this model classification is shown in Figure 2.

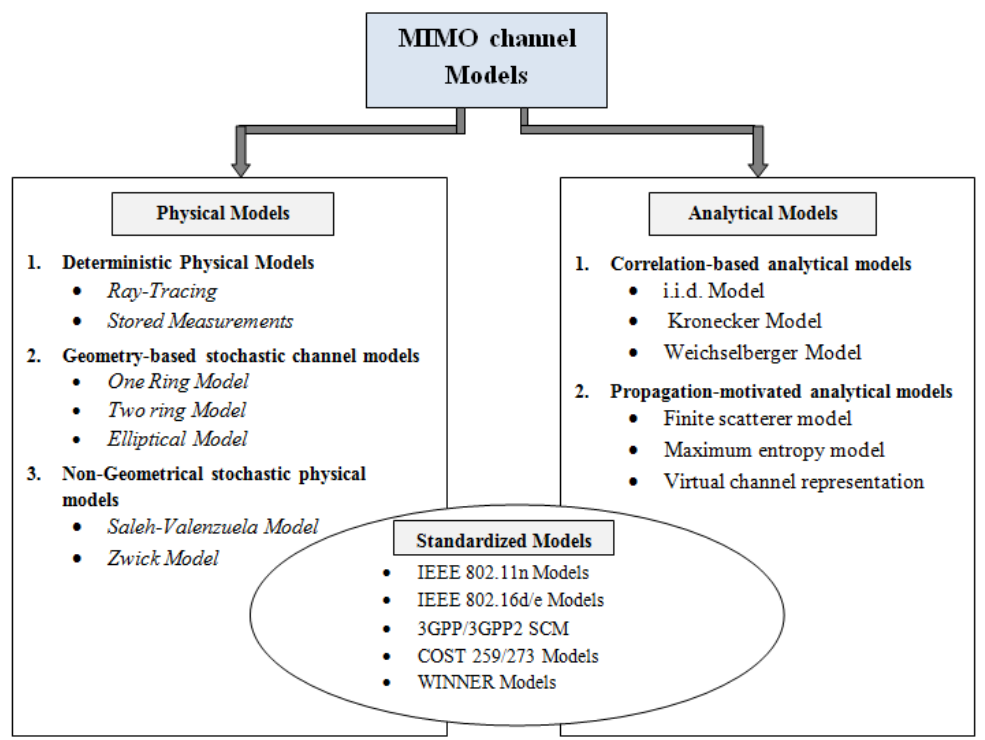

Figure 2. Classification of MIMO Channel Models

The MIMO channel models are distinguished as Physical and Analytical channel models, which are further divided into various models. The Deterministic model characterizes the parameters of physical propagation in a deterministic manner. The geometry-based stochastic channel models (GSCM) characterize the impulse response on the basis of wave propagation laws applied to specific transmitter, receiver and scatterer geometries, which are chosen in a stochastic (i.e. random) way. On the contrary, Nongeometric stochastic models depict the physical parameters like DoD, DoA, time delay, in a totally stochastic manner by prescribing the underlying probability distribution functions (PDFs) without assuming an underlying geometry. The Propagation-motivated models characterize the channel matrix via propagation parameters. Correlation-based models statistically describe the MIMO channel matrix in terms of the correlations between the matrix entries.

P. Almers and E. Bonek, [6], 2007, distinguish between the physical models and analytical models. It describes that the Physical channel models characterize an environment based on the electromagnetic wave propagation by considering the doubledirectional multipath propagation between the transmit $(\mathrm{Tx})$ and the receive $(\mathrm{Rx})$ array. The Physical channel models can be divided into Deterministic (for example: ray tracing and stored measurements); Geometry based stochastic (examples are one ring, two ring and elliptical) and Non-geometric stochastic models (for example: extensions of the Saleh-Valenzuela model). Analytical channel models characterize the impulse response of propagation channel between individual transmit and receive antennas in a mathematical (analytical) way without accounting for wave propagation. Analytical models are further subdivided into propagation-motivated models (for example: finite scatterer model, maximum entropy model, and virtual channel representation); and correlation-based models (examples: Kronecker model and Weichsel-berger model). This paper also illustrates the reference MIMO channel models defined by various organizations, known as Standardized models (examples are 3GPP, WINNER, COST 259/273, IEEE 802.16e, 
and IEEE 802.11n. As described in [10], the Physical channel models are mostly used for simulating system performance. However, they do not allow for the explicit design of space-time coding techniques. The Analytical models provide a mathematical representation of the channel matrix. A major difference between both types of modeling approaches lies in the fact that analytical models only provide a framework, but do not give fully quantitative information, unless they are parameterized by means of extrinsic information. Yet, analytical models are particularly useful when analyzing mathematically the impact of correlations on any performance parameter, since the relationship is explicit.

\subsection{Physical Models}

Physical channel models describe an environment on the basis of double-directional electromagnetic wave propagation between the location of transmit (Tx) array and the location of receive $(\mathrm{Rx})$ array. They model the wave propagation parameters like the Direction of Departure (DoD), Direction of Arrival (DoA), complex amplitude and delay of a Multi-Path Component (MPC) [6]. Physical models are independent of antenna configurations, such as number of antennas, antenna pattern, array geometry, polarization, mutual coupling; and system bandwidth. The Physical MIMO channel models can be subdivided into the deterministic models, the geometry-based stochastic models, and the non-geometric stochastic models.

4.1.1. Deterministic Physical Models: Deterministic models are aimed at reproducing the actual physical mobile propagation process for a given environment. Hence, deterministic channel models are physically significant, and potentially accurate. However, they are only representative for the environment considered. M. Patzold, [11], 2010, reviews that each methodology, deterministic or stochastic, has its own advantages and disadvantages. An advantage of the deterministic channel modeling approach is that the resulting simulation model is ergodic, while stochastic methods result always in nonergodic fading emulators. By using non-ergodic simulation models, several simulation runs has to perform with different sets of model parameters. On the other hand, if ergodic simulation models are employed, then a single or some few runs are sufficient in most cases. As a consequence, the performance of the majority of deterministic channel simulators can only be improved by increasing the number of sinusoids. The deterministic modeling method can be more suitable if the network planning or system level simulation has highly accurate requirements [12]. Examples of deterministic models are Ray Tracing and Stored Measurement Data [8].

\section{$>$ Ray-Tracing}

Ray Tracing method is used to model the propagation of electromagnetic wave through mobile communication channel. The size, location and the number of the reflectors; transmitters and absorbers are specified over time, and the accuracy the output results of ray tracing model depends on the availability of exact geometry in the database [6]. These methods have been widely applied to the mobile terrestrial channel. Indeed, as long as the wavelength is small compared to the dimensions of obstacles [11]. W. Jiang and K. Guan, [12], 2013, illustrates that the deterministic channel models based on Ray-tracing techniques, model the propagation channel using the geographical information from the database. The diffuse scattering is also taken into account on the surfaces seen by both the transmitter and receiver. Thus, all the surfaces of the structures in the scenario, for e.g. trees, buildings, vehicles, and traffic signs, are divided into tiles, and the single scattering processes are determined. For each ray, the channel polarization matrices are computed. M. A. Jensen and J. W. Wallace, [13] 2005 also reviewed that Ray tracing has emerged as the most popular technique for the analysis of site-specific scenarios, due to its ability to analyze very large structures with reasonable computational resources. 


\section{$>$ Stored Measurements}

R.B. Ertel and P. Cardieri [4], 1988, proposed a channel model based on the measurements. The idea behind this approach is to characterize the propagation environment, in terms of scattering points, based on measurement data. Measurement is used to determine the time-variant directional distribution of channel impulse response seen from the base station. This distribution is time- variant due to mobile motion and depends on the location, orientation, and velocity of the mobile station antenna and the topographical and morpho-graphical properties of the propagation area as well.

4.1.2. Geometry-Based Stochastic Channel Models (GSCM/ GBSCM): In deterministic geometrical approaches, the scatterer locations are given by a database. On the contrary, in geometry-based stochastic channel models (GSCM) the scatterer locations are choosen in a stochastic (random) manner according to a certain probability distribution [8]. Actual channel impulse response is then established by a simplified Ray Tracing procedure [6]. These models are useful for simulation and analysis purposes. Several important advantages of a GBSM are that it can be applied to physical/real conditions; as many effects are implicitly reproduced; all information is dependent on the distribution of the scatterers therefore, the model is not much complicated; transmitter/receiver and scatterer movement and the shadowing of propagation paths can be easily implemented. B. Chen and Z. Zhong, [14], 2012, propose the GBSCMs for terrain cutting, suburban, and urban scenarios in high-speed railway. With these channel models, the space correlation coefficients in several different scenarios are acquired. It shows that the antenna spacing has a huge impact on the space correlation. The GSCM urban and suburban channel models are also described in this paper. As described in [10], the GSCM models can be sub-divided into the regular-shaped GSCM (RS-GSCM) and the irregular-shaped GSCM (IS-GSCM), depending on whether the effective scatterers are distributed on a regular shape or irregular shape established by the propagation environment. The Regular Shaped GSCMs are divided into the following three models; that are one-ring model, two-ring model and elliptical model [15]:

\section{$>$ One-Ring Model}

The one-ring model is a popular geometry-based stochastic model. The One-ring model is preferred in modeling sub-urban environments [14], where the BS and MS are considerably distant from each other and the BS is at much higher height than the MS. Thus, it is assumed that the BS (transmitter) has little or no scatterers surrounding it, as may be placed on a very high point, for e.g. a hill, mountain or Tower; while the MS (receiver) is placed at a much lower point and is assumed to be surrounded by many scatterers existing on a ring around the MS. In Mobile-to-Mobile (M2M) communication systems, an example of this model may be an airplane or helicopter communicating with a ground vehicle. F.Z. Parizi and M. Mehrjoo [15], 2013, proposed one-ring, two-ring, and elliptical along with the ones for irregular shaped scattering environments, such as, streets and tunnels are reviewed extensively. The specification of each model in terms of appropriateness to be deployed for narrowband and/or wideband channels will be investigated. The cross-correlation function $(\mathrm{CCF})$ of the MS and the BS antenna elements is computed and then verified. Rachna M. and Jyoteesh M. [3], 2015, describes that the One-Ring Model assumes that the BS (transmitter) is not obstructed by any local scatterers, while the MS (receiver) is located at the center of a ring of surrounding by scatterers. This sort of assumption is suitable for propagation environments in the rural and the sub-urban areas, where high BS antennas are used. This paper analyzes the performance bounds of MIMO narrowband fading channels, i.e. One ring and Two ring channel models, assuming the non-line-of-sight (NLOS) propagation conditions from the transmission links from the MS to the BS. It provides a description of the temporal and spatial correlation properties of channel models along with their capacity. 


\section{$>$ Two-Ring Model}

The Two-ring model is similar to the One-ring model, where it is assumed that there is no LOS component and only local scatterers are considered. However, the difference is in the vertical position of the BS, which is not as higher as that in One-ring model. So, it is assumed that the BS is also surrounded by the scatterers existing on a ring along with the MS [3]. Therefore, the model has two rings, one surrounding the BS (which is the transmitter); and the other surrounding the MS (which is the receiver) where the scatterers exists [10]. In Mobile-to-Mobile communications, the two ring model may be seen as a helicopter flying low in the city communicating with a ground vehicle. The paper [15] also illustrates the design and performance analysis of MIMO channel model. A general procedure is proposed for the designing of stochastic and deterministic simulation models which intended for the frequency-nonselective MIMO Rayleigh fading channels based on the geometrical two-ring scattering model. S. Wang and A. Abdi, [16], 2005, proposed a single-bounce two-ring statistical model for time-varying MIMO flat fading channels. The model includes some key physical channel parameters such as the mean angle-ofdeparture (AOD) and angle-of-arrival (AOA), angle spreads, maximum Doppler frequency, sizes of the local scattering rings, and the relative contribution of BS and MS local scatterers. Comparison of the model with outdoor and indoor collected data, in terms of spatial correlations, temporal autocorrelations, spatio-temporal cross-correlations, level crossing rates, average fade duration, and the distribution of mutual information has demonstrated the flexibility of the model in describing real-world propagation environments. The proposed model, supported by empirical observation, provides a useful channel characterization required for efficient design and performance prediction of multi-antenna transceivers.

\section{$>$ Elliptical Model}

The Elliptical Model is designed for wideband MIMO channel modeling with typical temporal, spatial, and frequency correlation properties as well, in contrast to the one-ring and two-ring models. The most important feature of elliptical models is frequency selectivity that makes it a very attractive model for the developers of future wireless mobile communication systems, as the designing, analysis and performance investigation of high data rate wireless communication systems employing MIMO-OFDM techniques, has gained a huge interest. Geometry-based elliptical model [15] assumes that scatterers are uniformly distributed within an ellipse where the MS and BS are the foci of the ellipse for wideband modeling, multiple ellipses surround the MS and the BS. The MS and BS are assumed to have equal antenna heights in this model. G. Tsoulos [17], 2006, illustrates that elliptical model produces a high probability of scatterers with minimum excess delay along the LOS. It also briefly explains the Elliptical Subregions Model, where the ellipse is subdivided into a number of elliptical subregions and Elliptical Model with Dense Discrete Scatterers, where the scatterers are distributed on ellipsoids. X. Cheng and C.X. Wang [18], 2009 proposed a generic and adaptive GBSM for non-isotropic MIMO mobile-to-mobile (M2M) fading channels. The model proposed employs a combined model of elliptical and two-ring, with the received signal is the sum of LOS, single and double-bounced rays with different energies. Thus the model is sufficiently generic and adaptable to a various M2M scenarios, such as macro, micro and pico-cells.

4.1.3. Non-Geometrical Stochastic Physical Models: The non-geometric stochastic models (or Empirical models) describes and determines the physical parameters like DoD, DoA or delay, in a completely stochastic manner by specifying the underlying pdf (probability distribution functions) without assuming the geometry of physical environment. The stochastic non-geometrical models have two types reported in the literature. The first type uses clusters of MPCs known as extended Saleh-Valenzuela (SV) model as it is a generalization of the temporal 
cluster model developed in [19]. The second type individually treats the multipath components (MPCs), and is known as Zwick model [6].

\section{$>$ Saleh-Valenzuela Model}

A. A. M. Saleh and R. A. Valenzuela, [19], 1987, proposed a channel model, called Saleh and Valenzuela (SV), to model clusters of MPCs in the delay domain through a doubly exponential decay process. An exponentially decaying profile is used in this model to control and manage the power of a multipath cluster. The SV model has been extended to the spatial domain. Particularly, this extended SV-MIMO model assumes that the DoD and the DoA statistics are independent and identical. K.V. Anusuya and S. Bharadhwaj, [20], 2008, describe that the SV model is based on the physical realization that the received signal rays arrive in clusters, with each cluster comprising several rays. This model developed a multipath channel model for indoor environment based on the clustering phenomenon, which refers to the observation that multipath components arrive at the antenna in groups. In [17] the SV model given for indoor environments employs the clustered "double Poisson" time-of-arrival model, with statistical independence between time and angle. The mean angles of each cluster are distributed uniformly over all angles. In [10] the multipath components are experimentally found to arrive in groups, or clusters of scatterers. It is further observed that clustering also takes place in the angular domain.

\section{$>$ Zwick Model}

As it is analyzed in paper [6], that clustering and multipath fading for the indoor channels does not occur when the sampling rate is sufficiently large. Hence, Zwick model generates the MPCs, independently and without amplitude fading. However, the phase changes of MPCs are included into the model on considering the geometric descriptions of the motion of Tx, Rx and scatterer.

\subsection{Analytical Models}

In contrast to physical models, Analytical channel models characterizes the channel impulse response or the transfer function between transmit and receive antennas individually in a mathematical analytical manner without accounting for wave propagation explicitly. The individual impulse responses are considered as MIMO channel matrix. These channel models are widely used in system simulations because they are relatively easy to generate and can be used to represent various types of channel responses [6]. There is no direct correspondence between the channel matrix and the actual physical environment in which the MIMO system is operating. The antenna type used heavily affects the channel model. Analytical models can be further subdivided into Correlation-based models and Propagation- motivated models.

\subsubsection{Correlation-Based Analytical Models}

A Correlation-based analytical model statistically characterizes the MIMO channel matrix in terms of the correlations between the matrix entries. Most commonly used correlation-based analytical channel models are the I.I.D(independent and identically distributed) model, Kronecker model and the Weichsel Berger model.

\section{$>$ The I.I.D(Independent And Identically Distributed) Models}

The I.I.D. model the simplest analytical MIMO model, which is sometimes also referred to as canonical model. This model consists of a single parameter only and is often used for information theoretic analysis of MIMO systems and other theoretical considerations [6]. A range of narrowband analytical models are based on the multivariate 
complex Gaussian distribution of MIMO channel coefficients; that is Rayleigh or Ricean fading. In [10] it is presented that as in the case of single-antenna channels, the Rayleigh fading assumption is often used by MIMO system designers, mostly because it is realistic in environments rich in scatterers. The I.I.DRayleigh assumption has been used extensively when designing space-time codes. K. Zheng and O. Suling [21], 2014, present that the I.I.DRayleigh fading channel model is extensively adopted for the theoretical analysis of massive MIMO systems, where it is assumed that no correlation and mutual coupling is there between transmit antennas or receiver antennas. This favorable propagation is the most imperative property of the I.I.DRayleigh fading channel in the MIMO system.

\section{> The Kronecker Model}

The Kronecker model simplifies the expression of the full correlation matrix by using a separability assumption. In this model it is assumed that the spatial $\mathrm{Tx}$ and $\mathrm{Rx}$ correlations are separable, which is correspondent to limiting the correlation matrices. The specifications of the $\mathrm{Tx}$ and $\mathrm{Rx}$ correlation matrices for this model. Moreover, an independent array optimization at $\mathrm{Tx}$ and $\mathrm{Rx}$ is allowed by the underlying separability of $\mathrm{Tx}$ and $\mathrm{Rx}$ in the Kronecker sense. The Kronecker model has become quite popular due to these applications and its simplicity [6]. C. Zimmermann and M. Paasch, [22], 2007, compare the two existing stochastic MIMO channel models, i.e. Kronecker model and Weichselberger. The assumption of the Kronecker model is that the correlations of the MIMO channel can be separated into two correlation matrices, one for the correlations at transmitter site and one for the correlations at the receiver site. The Weichselberger model relaxes the Kronecker model assumption by implying constant eigenvectors for both link ends. The results show that in contrast to the Kronecker model, the outage capacity of the Weichselberger model exhibits the expected conduct concerning the ideal characteristics.

\section{$>$ The Weichselberger Model}

The Weichselberger model intends to prevent the restrictions of the Kronecker model to a separable DoA-DoD spectrum that ignores the significant parts of spatial configuration of MIMO channels [6]. B. Ernst, [23], 2006, illustrates that the Weichselberger model is a generalization of MISO eigenmode analysis to MIMO. The main assumption is that the spatial eigenbases are not affected by the transmit weights and, thus, reflect the radio environment only, i.e. number, positions, and strengths of the scatterers. Paper [8] proposed that to derive this model, it is importance for the Kronecker model to understand the coupling between the transmit Tx and receives Rx eigen modes. So, the parameters for the Weichselberger model represent the coupling matrix, and the eigen bases of the transmit and receive correlation matrices.

\subsubsection{Propagation-Motivated Analytical Models}

Propagation-motivated models describe the MIMO channel matrix via propagation parameters. Examples of such analytical models are the Finite Scatterer Model, Maximum Entropy Model, and Virtual Channel Representation.

\section{$>$ Finite Scatterer Model}

The finite scatterer model basically assumes that the propagation channel can be modeled in terms of a finite number of multipath components. This model allows for the single-bounce and multiple-bounce scattering, which is in contrast to GSCMs that usually only incorporate single-bounce and double-bounce scattering [6]. F.P. Fontan and P. M. Espineira, [24], 2008, simulate the MIMO channel using the multiple point-scatterer approach, and it is shown how the capacity can be increased substantially. Three models are proposed based on the three projects, which are generating clustered point-scatterer 
propagation scenarios, MIMO channel modeling using the multiple point scatterers and statistical modeling of the MIMO channel.

\section{$>$ Maximum Entropy Model}

The maximum entropy principle was proposed to determine the distribution of the MIMO channel matrix on the basis of prior information available. This priori information may contain the properties of propagation environment and system parameters, like bandwidth and DoAs. The principle of maximum entropy is justified by the objective to avoid the model assumptions which do not support earlier information [6]. Different channels models can be obtained for the capacity calculations than for bit error rate (BER) simulations.

\section{$>$ Virtual Channel Representation}

C. Oestges and B. Clerckx, [10], 2007, propose that the original idea of virtual model is to relax the correlation-separability restriction of the Kronecker model and to allow for any arbitrary coupling between the transmit and receive ends of the link, while preserving a relative ease-of-use. This model can be viewed as a special case of the Weichselberger model with Tx and Rx eigenmodes equal to the columns of DFT matrices. The virtual channel model can be reduced to the I.I.Dchannel model, means a rich scattering with complete links of Tx and Rx scatterer clusters. The virtual channel model is typically useful for theoretical considerations due to its simplicity, like for analyzing the capacity scaling performance of MIMO channels [6]. H. Ozcelik and N. Czink, [2], 2006, describes that the virtual channel representation (VCR) models the MIMO channel in the beamspace instead of the eigenspace. In particular, the eigen-vectors are replaced by fixed and predefined steering vectors and the VCR can be easily interpreted. The angular resolution of VSR, and hence the accuracy, depends on the actual antenna configuration. Its accuracy increases with the number of antennas, as the angular bins become smaller.

\subsection{Standardized Models}

When comparing the performance of different system implementations, it is convenient to use Standardized models established by several international organizations [10]. Thus, Standardized models are an essential tool for the development of new radio systems. They allow assessing the benefits of different techniques for capacity and performance enhancement, in a unified manner agreed on by many parties [6]. With physical models, it specifies channel model, reference environments, and their parameter values. And with analytical models, the parameter sets represent for the target scenarios. Examples for models are 3GPP SCM, WINNER, COST 259/273, IEEE 802.16a,e, and IEEE 802.11n. In this section, these standardized MIMO channel models are discussed to provide an overview of recent and ongoing channel modeling activities.

\section{$>$ IEEE 802.11n Channel Models}

This set of models is an improved and standardized version of the extended SalehValenzuela model, with overlapping clusters in the delay domain. The models have been designed for indoor MIMO wireless local area networks at both 2 and $5 \mathrm{GHz}$ and for bandwidths up to $100 \mathrm{MHz}$ [6]. Six canonical channels are modeled, covering flat fading, residential, small office, typical office, large office and large open spaces [10].

\section{$>$ IEEE 802.16d/e Channel Models}

IEEE 802.16d/e models are intended for macrocellular fixed wireless access. In fact, IEEE 802.16 models are the improved versions of SUI (Stanford University Interim) channel models, which is valid for both omni-directional and directional antennas. The use of directional antennas will cause the global K-factor to increase while the delayspread will decrease [10]. S. C. Yong and K. Jaekwon, [7], 2010, explain the IEEE 
802.16d model is based on the log-normal shadowing path loss model. There are three different types of models (Type A, B, and C), depending on the density of obstruction between the transmitter and receiver (in terms of tree densities) in a macro-cell suburban area. D. Klaus [25], 2008, describes that in IEEE 802.16e standard, the MIMO channel models are specified in WiMax Forum. IEEE $802.16 \mathrm{~m}$ has initiated and several channel model contributions has been submitted.

\section{> 3GPP/3GPP2 Spatial Channel Models (SCM)}

The 3GPP/3GPP2 channel models are established specifically for the simulation of third generation networks in urban and suburban macrocells as well as in urban microcells. The modeling structure is similar to that of the COST 259 directional channel model [10]. F. Darbari and R.W. Stewart, [5], 2010, describes that the Spatial Channel Model (SCM) is developed by $3 \mathrm{GPP} / 3 \mathrm{GPP} 2$ to provide a common reference for evaluating different concepts of MIMO in outdoor environments at $2 \mathrm{GHz}$ center frequency and $5 \mathrm{Mz}$ system bandwidth. The SCM consists of two parts; a calibration model, and a system-simulation model. Moreover, the SCM system level model is currently used as a de-facto standard for LTE, WCDMA, UMTS and WiMAX system level evaluation and performance verification.

\section{$>$ COST 259/273}

"COST" is an abbreviation for European Cooperation in the field of Scientific and Technical research. Various COST initiatives were dedicated to wireless communications, in particular COST 259 "Flexible personalized wireless communications" (1996-2000) and COST 273 "Towards mobile broad- band multimedia networks" (2001-2005) [6]. They are a general standardized channel models, and are not proposed for specific systems. J. Poutanen [26] represents that the COST 2100 MIMO channel model is also a development of the geometry-based stochastic channel models (GBSM) developed by the COST actions.

The COST 259 directional channel model (DCM) was originally established for the simulation of systems with multiple antenna elements at either the BS or the MS. The model describes the joint impacts of small-scale as well as large-scale effects, and covers macro, micro and pico-cellular scenarios [10]. The COST 273 channel model shows considerable similarity to the COST 259 model, however differs in several key respects. As a number of new radio environments are defined, reflecting the new applications for MIMO systems, like peer- to-peer and the fixed-wireless access scenarios. As compared to the COST 259 model, modeling of the distribution of DOAs and DODs is different [6].

\section{$>$ SUI (Stanford University Interim) Channel Models}

The Stanford University Interim (SUI) channel models were developed for the macrocellular fixed wireless access networks operating at $2.5 \mathrm{GHz}$ and were further enhanced in the framework of the IEEE 802.16a standard [6]. They are basically tapdelay line models, with assigned envelope antenna correlation coefficients. Antennas are assumed to be omni-directional at both sides [17].

\section{$>$ WINNER Channel Models}

The European WINNER (wireless world initiative new radio) project began in 2004 with the aim to develop a new radio concept for beyond third generation (B3G) wireless systems [5]. Paper [6] describes that the channel models developed in the IST-WINNER project are associated with both the COST 259 model and the 3GPP SCM model. The novel features of the model are its parameterization, the consideration of elevation in indoor scenarios, autocorrelation modeling of large-scale parameters and scenariodependent polarization modeling. The model can be scaled from a single SISO or MIMO 
link to a multilink MIMO scenario including polarization among other radio channel dimensions.

\section{Issues and Challenges}

Based on literature survey done in the previous section, various issues and challenges have been extracted and tabulated in Table 1:

Table 1. Issues and Challenges for Different MIMO Channel Models

\begin{tabular}{|c|c|c|c|}
\hline er ${ }^{\text {Pap }}$ & \begin{tabular}{|c|} 
Channel \\
Model
\end{tabular} & Description & Issue \\
\hline \multirow[t]{2}{*}{$\begin{array}{l}\text { Richa } \\
\text { d B. Ert } \\
\text { and Paul } \\
\text { Cardieri } \\
\text { [4], 1988 }\end{array}$} & $\begin{array}{l}\text { Geometrical } \\
\text { B Based Single } \\
\text { Bounce } \\
\text { Statistical } \\
\text { Models }\end{array}$ & $\begin{array}{l}\text { These channel models } \\
\text { are defined by spatial } \\
\text { scatterer density function, } \\
\text { from which the joint and } \\
\text { marginal TOA and AOA } \\
\text { pdf are derived. }\end{array}$ & $\begin{array}{l}\text { To provide an accurate channel } \\
\text { model, the shape and size of the } \\
\text { spatial scatterer density function are } \\
\text { required. }\end{array}$ \\
\hline & $\begin{array}{c}\text { Ray } \\
\text { Tracing }\end{array}$ & $\begin{array}{l}\text { Ray tracing model is an } \\
\text { entirely site-specific models } \\
\text { which has the potential to be } \\
\text { extremely accurate. }\end{array}$ & $\begin{array}{l}\text { A comprehensive description of } \\
\text { the physical propagation } \\
\text { environment and measurements is } \\
\text { required to validate the models. }\end{array}$ \\
\hline $\begin{array}{l}\text { George } \\
\text { Tsoulos } \\
{[17], 2006}\end{array}$ & $\begin{array}{l}\text { Determinis } \\
\text { ic Models }\end{array}$ & $\begin{array}{l}\text { The deterministic } \\
\text { propagation modeling is } \\
\text { described with Ray-Tracing. }\end{array}$ & $\begin{array}{l}\text { An efficient computational } \\
\text { technique to trace back the paths } \\
\text { between the Tx and Rx rapidly and } \\
\text { accurate knowledge of electrical } \\
\text { characteristic parameters for all } \\
\text { obstacles is required. }\end{array}$ \\
\hline $\begin{array}{l}\text { Huseyin } \\
\text { Ozcelik } \\
{[2], 2006}\end{array}$ & $\begin{array}{l}\text { Analytical } \\
\text { Models }\end{array}$ & \begin{tabular}{l}
\multicolumn{1}{c}{ The accuracy of } \\
analytical MIMO channel \\
models (Kronecker model \\
and virtual channel \\
representation) \\
investigated using different \\
meaningful measures of \\
quality.
\end{tabular} & $\begin{array}{l}\text { The Kronecker model can be } \\
\text { used for limited antenna numbers } \\
\text { only, and the virtual channel } \\
\text { representation can be used for } \\
\text { modeling the joint angular power } \\
\text { spectrum only, for very large } \\
\text { antenna numbers. }\end{array}$ \\
\hline $\begin{array}{l}\text { Peter } \\
\text { Almers, } \\
\text { Ernst } \\
\text { Bonek, } \\
{[6], 2007}\end{array}$ & COST 259 & $\begin{array}{l}\text { The model describes the } \\
\text { joint impacts of small-scale } \\
\text { as well as large-scale } \\
\text { effects, and illustrates the } \\
\text { macro, micro and } \\
\text { picocellular scenarios. }\end{array}$ & $\begin{array}{l}\text { The scatterers are assumed to be } \\
\text { stationary in order to investigate the } \\
\text { channel time variations due to MS } \\
\text { movement only. Hence certain } \\
\text { environments, such as; indoor } \\
\text { scenarios with persons moving } \\
\text { around cannot be included. }\end{array}$ \\
\hline $\begin{array}{l}\text { Christian } \\
\text { Zimmer } \\
\text { mann } \\
{[22]} \\
2007\end{array}$ & $\begin{array}{l}\text { Kronecker } \\
\text { and } \\
\text { Weichselber } \\
\text { ger model }\end{array}$ & $\begin{array}{l}\text { Paper compares the } \\
\text { existing stochastic MIMO } \\
\text { channel models with an } \\
\text { existing laboratory MIMO } \\
\text { system. }\end{array}$ & $\begin{array}{l}\text { The Kronecker model provides } \\
\text { the worst results. The } \\
\text { Weichselberger model also lags the } \\
\text { accuracy in MIMO channel model } \\
\text { and its capacity. }\end{array}$ \\
\hline $\begin{array}{c}\text { David } \\
\text { Klaus } \\
{[25]} \\
2008\end{array}$ & $\begin{array}{l}\text { Standardized } \\
\text { models }\end{array}$ & $\begin{array}{l}\text { In the standardization of } \\
\text { MIMO channel models, the } \\
\text { realistic modeling and } \\
\text { computational complexity } \\
\text { requirements } \\
\text { inconsistent, when they used } \\
\text { for system-level } \\
\text { performance evaluation with } \\
\text { hardware or software } \\
\text { simulations. }\end{array}$ & $\begin{array}{l}\text { The simulation time has to be } \\
\text { reduced to keep the models simple. } \\
\text { A backward compatibility is often } \\
\text { required to keep the new results } \\
\text { comparable to the old ones. }\end{array}$ \\
\hline
\end{tabular}




\begin{tabular}{|c|c|c|c|}
\hline \multirow[t]{2}{*}{$\begin{array}{l}\text { Wenyi } \\
\text { Jiang } \\
\text { and Ke } \\
\text { Guan } \\
\text { [12], } \\
2013\end{array}$} & $\begin{array}{l}\text { Deterministic } \\
\text { channel } \\
\text { models }\end{array}$ & $\begin{array}{l}\text { These models propose } \\
\text { the highly accurate } \\
\text { simulation of the realistic } \\
\text { channel model including the } \\
\text { non-stationarity of the } \\
\text { channel naturally. }\end{array}$ & $\begin{array}{l}\text { They need highly accurate } \\
\text { topographical databases, and are } \\
\text { numerically intensive to process. } \\
\text { Also, for Ray-tracing method, the } \\
\text { high resolution digital elevation } \\
\text { model is very expensive. }\end{array}$ \\
\hline & $\begin{array}{l}\text { Stochastic } \\
\text { and } \\
\text { Geometry- } \\
\text { based } \\
\text { stochastic } \\
\text { models } \\
\text { (GBSM) }\end{array}$ & $\begin{array}{l}\text { Stochastic } \\
\text { particularly the tapped delay } \\
\text { line models can be } \\
\text { parameterized in a relatively } \\
\text { flexible way to describe } \\
\text { channels in different } \\
\text { scenarios. Geometry-based } \\
\text { stochastic modeled are uses } \\
\text { a simplified ray tracing } \\
\text { along with random } \\
\text { scatterers. }\end{array}$ & $\begin{array}{l}\text { The stochastic tapped delay line } \\
\text { models do not consider the non- } \\
\text { stationarity of the channel. } \\
\text { Therefore, more research efforts are } \\
\text { required to introduce the non- } \\
\text { stationarity to the stochastic channel } \\
\text { models. GBSM can completely } \\
\text { describe the non-stationarity of the } \\
\text { channel. But they require more } \\
\text { computation time than stochastic } \\
\text { channel models. }\end{array}$ \\
\hline
\end{tabular}

\section{Open Issues and Future Scope}

A lot of research has been reported in the literature related to the MIMO channel modeling in the mobile wireless systems. Still, the present issue is to "make the thing work' in real-world wireless systems and under realistic power constraints. From the literature survey done in the section 5, it has been observed that there is an ample scope for the system designers and researchers to work in this direction. Various open challenges that need attention from the research community are enumerated below:

One of the main open issues in the existing geometry-based stochastic channel models is the incorporation of the so called dense multipath components in the models. A serious effort must be put in adding them to the existing implementations of the GSCMs. The pragmatic extensions of physical models to the multiple clusters of scatterers and to the frequency selectivity models are another open works. Also, for deterministic channel models, an up-to-date high-resolution database has to be available. In addition, modeling of diffuse scattering is necessary. However, identification and modeling of the spatial structure of diffuse scattering is still an open task which needs further research.

The existing channel models and measurement procedures do not adequately consider 3D (three dimensional) nature of radio wave propagation. Hence, there are still numerous open issues in developing realistic multi-dimensional channel models for future wireless communication systems. These include but are not limited to complexity intercell interference modeling and, stationarity and scale effects.

Significant advances have been made in the area of MIMO channel modeling for mobile wireless systems. Yet, a number of MIMO techniques still have not been effectively tested under realistic channel propagation conditions. Consequently their integration into practical applications can be considered to be still in its infancy. Nevertheless, a number of effects known from measurements are still there, which are not considered reliably in the existing models. Some of these features lacking in current models are keyhole effect, diffuse multipath components, polarization and time variation. The parameterization of appropriate models for emerging new scenarios, like outdoor-toindoor, and distributed MIMO networks is still an open issue. Besides, the increased number of features and improved accuracy lead to more specialized and complex models. Therefore, the validation of these channel models through extensive measurements remains an active area of research. Consequently, more effort is necessary to validate channel models and to determine the applicability of the models in different environments. 


\section{Conclusion}

Channel model describes the propagation characteristics of signals in mobile communication environments and are very critical for the performance evaluation of wireless communication systems. Thus it is incredibly important to study and investigate the state of the art in MIMO channel models for the wireless communication systems. The purpose of this paper is to provide an overview of the most significant concepts in the modeling of MIMO channels for the mobile wireless systems. Starting from different classifications of MIMO channel models, the physical, analytical models and standardized channel models are studied. The physical model focuses on double-directional propagation without considering the antenna characteristics and analytical models concentrates on the channel impulse response including antenna properties. The standardized models are defined by various organizations as the reference models for different wireless standards. For all these model types, the popular examples are reviewed that are widely used for the design and evaluation of MIMO systems. Based on the survey made in this paper, some problems and issues are extracted and finally some open issues are taken out which need to be resolved in the future.

\section{References}

[1] M. Rachna and M. Jyoteesh, IEEE International Conference On Advances In Engineering \& Technology Research, (2014).

[2] H. Ozcelik, N. Czink and E. Bonek, IEEE Vehicular Technology Conference, (2006).

[3] M. Rachna and M. Jyoteesh, International Journal of Computers \& Technology, Ijct Cir World, (2015).

[4] R. B. Ertel and P. Cardieri, IEEE Personal Communications, (1998).

[5] F. Darbari, R. W. Stewart and I. A. Glover, "Signal Processing", Intech., (2010).

[6] P. Almers, E. Bonek, D. I. Laurenson and H. Ozcelik, "Eurasip Journal On Wireless Communications And Networking", Hindawi Publishing Corporation (2007).

[7] S. C. Yong, K. Jaekwon and G. K. Chung, IEEE Press, John Wiley \& Sons (2010)

[8] W. K. Saad, M. Ismail and R. Nordin, Journal Of Communications, Academy Publisher (2012)

[9] N. Costa and S. Haykin, "Multiple-Input, Multiple-Output Channel Models Theory And Practice", Published By John Wiley \& Sons (2010)

[10] C. Oestges and B. Clerckx, "Mimo Wireless Communications, Academic Press", Elsevier, (2007)

[11] M. Patzold, "Mobile Radio Channel", Second Edition, John Wiley \& Sons, Ltd. (2010)

[12] W. Jiang, K. Guan, Z. Zhong and J. You, International Journal Of Antennas And Propagation, Hindawi Publishing Corporation (2013)

[13] M.A. Jensen and J. W. Wallace, "Space-Time Processing For Mimo Communications", (2005) John Wiley \& Sons, Ltd

[14] B. Chen and Z. Zhong, International Journal Of Antennas And Propagation, Hindawi Publishing Corporation (2012)

[15] F.Z. Parizi, M. Mehrjoo and J.A. Shokouh, Technical Journal Of Engineering And Applied Sciences (2013)

[16] S. Wang, A. Abdi, J. W. Wallace and M. A. Jensen, IEEE International Workshop On Signal Processing Advances For Wireless Communications (2005)

[17] G. Tsoulos, "Mimo System Technology For Wireless Communications", CRC Press, Taylor \& Francis Group, (2006).

[18] X. Cheng, C. X. Wang, D. I. Laurenson and A. V. Vasilakos, IEEE Transactions On Wireless Communications, (2009).

[19] A. M. Saleh and R. A. Valenzuela, IEEE Journal on Selected Areas In Communications, (1987).

[20] K. V. Anusuya, S. Bharadhwaj and S. Subha, Defence Science Journal, (2008).

[21] K. Zheng, O. Suling and Y. Xuefeng, International Journal Of Antennas And Propagation, Hindawi Publishing Corporation, (2014).

[22] C, Zimmermann, M. Paasch, O. Weikert and U. Zolzer, "On Investigating Spatial Correlations of Mimo Indoor Channels", Proc. 12th International Ofdm-Workshop, Germany, August 29-30.

[23] B. Ernst, "The Mimo Radio Channel”, Technische Universität Wien, Vienna, Austria, (2006).

[24] F. Perez Fontan and P. Marino Espineir, "Modeling The Wireless Propagation Channel A Simulation Approach With Matlab", John Wiley \& Sons Ltd Publications, (2008).

[25] D. Klaus, "Technologies for the Wireless Future: Wireless World Research Forum", John Wiley and Sons, (2008) September.

[26] J. Poutanen, K. Haneda, C. Oestges and P. Vainikainen, European Conference on Antennas and Configurations, (2011). 


\section{Authors}

Rachna Mahey was born in Punjab, India. She received her B.Tech. in 2013; and M.Tech in 2015 from Electronics and Communications Department, Guru Nanak Dev University, Regional Campus, Jalandhar, India. Her research areas include Performance Characterization of Fading Channels and MIMO wireless systems.

Dr. Jyoteesh Malhotra B.Eng., M.Tech, Ph.D. is involved in teaching and research at Electronics and Communications Department, Guru Nanak Dev University, Regional Campus, Jalandhar, India. His research areas of interest include Statistical modelling of Fading Channels and Fading mitigation techniques in Wireless Communication. Dr. Malhotra has more than 100 research publications and authored 02 books. He is a life member of I.S.T.E. and editorial board of many International Journals of repute. 
International Journal of Future Generation Communication and Networking Vol. 8, No. 5 (2015) 